\title{
Capillarity in porous media: Recent advances and challenges
}

\author{
Jianchao Cai ${ }^{1, *}$ (D), Pål Ø. Andersen ${ }^{2}$ (D), and Shuangmei Zou ${ }^{3}$ (B) \\ ${ }^{1}$ State Key Laboratory of Petroleum Resources and Prospecting, China University of Petroleum, Beijing 102249, PR China \\ ${ }^{2}$ Department of Energy Resources, University of Stavanger, Kjell Arholms Gate 41, 4036 Stavanger, Norway \\ ${ }^{3}$ Key Laboratory of Tectonics and Petroleum Resources, Ministry of Education, China University of Geosciences, \\ Wuhan 430074, PR China
}

Accepted: 28 October 2021

Capillarity refers to the phenomenon of fluids imbibing into fine pores or porous rocks and materials due to surface energy, through the action of capillary pressure. The capillary pressure causes the deformable interfaces formed between two phase fluids in contact with each other, such as a liquid and a vapor.

Capillarity and its importance have been widely recognized in various fields, including civil engineering, soil science, hydrology, carbon storage, and petroleum engineering. More than a century ago, the classical Young-Laplace equation and Lucas-Washburn equation laid the foundation for the description of the capillary-driven flow of fluids in porous structures.

With the rapid development of computational and experimental techniques, the research on capillarity is advancing at an unprecedented level and provides insight and opportunities for further effort in what is already a flourishing field of research.

Four papers on capillarity in porous media are collected in this special issue and are summarized in the following paragraphs:

In the paper "Shale adhesion force measurements via atomic force microscopy" [1], Mitiurev et al. used the AFM (Atomic Force Microscopy) technique to measure the adhesion force between cantilever and sample surface at nanoscale for shale. They used topographical analysis, SEM (Scanning Electron Microscopy) and EDS (Energy Dispersive Spectroscopy) to accurately locate the measurement points on a sample surface and obtained consistent adhesion force measurements for quartz grain inclusions. The adhesion forces were determined to equal the waterair capillary pressure under the test conditions. These measurements and results have potential to create a path towards much higher accuracy-wettability measurements and consequently better reservoir-scale predictions and improved underground operations.

In the paper "A modified cell-to-cell simulation model to determine the minimum miscibility pressure in tight/shale

\footnotetext{
* Corresponding author: caijc@cup.edu.cn
}

formations" [2], Sun and Li developed a new oil-gas minimum miscibility pressure calculation algorithm, which couples the effects of capillary pressure and confinement in the original cell-to-cell simulation model to predict the oil-gas minimum miscibility pressure in a confined space. By the algorithm, the effects of temperature, pore radius, and injection gas impurity on the confined oil gas minimum miscibility pressure calculations are studied in detail. They found that the oil gas minimum miscibility pressure is reduced in confined space and the degree of this reduction depends on the pore radius. For all the tested pore radii, the confined minimum miscibility pressure first increases and then decreases with an increasing temperature. Compared to pure carbon dioxide injection, the addition of methane in the injection gas increases the oil gas minimum miscibility pressure in confined nanopores. It is recommended to control the content of methane in the injection gas in order to achieve a more efficient gas injection design.

In the paper "Capillarity and phase-mobility of a hydrocarbon gas-liquid system" [3], Gao et al. applied X-ray computed microtomography to image the pore level gas and liquid distribution in 3D while lowering the pressure below the bubble point or while injecting gas. The pore level gas and liquid distribution gives direct access to the connectivity of the gas and hence its mobility. Respective (connected pathway) relative permeability is directly computed from the imaged gas and liquid distribution using lattice Boltzmann-based flow simulation. The gas relative permeability follows a classical non-wetting phase drainage relative permeability curve when re-scaling the saturation endpoint to the critical gas saturation. The liquid relative permeability is compatible with both a wetting phase imbibition and drainage relative permeability. Their study provides fundamental insight into the gas dynamics in two-component systems that fall below the bubble point pressure and gas is nucleated.

In the paper "Analytical modeling and correction of steady state relative permeability experiments with capillary end effects - An improved intercept method, scaling and general capillary numbers" [4], Andersen presented 
general analytical solutions for calculation of spatial saturation and pressure gradient profiles, average saturation, pressure drop, and relative permeabilities for a core at steady state when capillary end effects are significant. He derived an intuitive and general intercept method for correcting steady state relative permeability measurements for capillary end effects: plotting average saturation and inverse effective relative permeability (of each phase) against inverse total rate will give linear trends at high total rates and result in corrected relative permeability points when extrapolated to zero inverse total rate (infinite rate). The findings of this work are relevant to accurately estimate relative permeabilities in steady state experiments, relative permeability end points and critical saturations during flooding or the impact of injection chemicals on mobilizing residual phase.

The study of capillarity still faces many problems, such as scale. Capillarity controls fluid flow, trapping, and distribution in porous networks and can be characterized most accurately on micro- and nanometer levels, but often must be modeled macroscopically due to the scales of systems where fluid flow is relevant, such as geological subsurface formations. At that scale fluids are treated as a continuum. However, as the systems under study become smaller, approaching the nanometer scale, such an approximation is expected to break down. Many phenomena at nanoscale are not easily upscaled to continuum approaches. Indeed, numerous experimental and computational studies indicate that at the nanoscale, the molecular nature of liquids cannot be avoided. In addition, the multi-physical mechanisms, structural, and compositional heterogeneity of porous media also play an important role in capillarity. At present, their combined effect still remains unclear. Not surprisingly, the research for capillarity is and will be a hot topic for further research.
Acknowledgments. The guest editors would like to thank the authors for their contributions to the special issue and the reviewers for their constructive comments. We also thank the editorial and administrative staff of Oil \& Gas Science and Technology for facilitating the special issue. Cai acknowledges the National Natural Science Foundation of China and the Fundamental Research Funds for the Central Universities for supporting his series of studies on flow and transport in porous media. Andersen acknowledges the Research Council of Norway and the industry partners, ConocoPhillips Skandinavia AS, Aker BP ASA, Vår Energi AS, Equinor Energy AS, Neptune Energy Norge AS, Lundin Energy Norway AS, Halliburton AS, Schlumberger Norge $A S$, and Wintershall Dea Norge AS, of The National IOR Centre of Norway for support.

\section{References}

1 Mitiurev N., Verrall M., Shilobreeva S., Keshavarz A., Iglauer S. (2021) Shale adhesion force measurements via atomic force microscopy, Oil Gas Sci. Technol. - Rev. IFP Energies Nouvelles 76, 73.

2 Sun H., Li H. (2021) A modified cell-to-cell simulation model to determine the minimum miscibility pressure in tight/shale formations, Oil Gas Sci. Technol. - Rev. IFP Energies nouvelles 76, 48.

3 Gao Y., Georgiadis A., Brussee N., Coorn A., van der Linde H., Dietderich J., Alpak F.O., Eriksen D., Mooijer-van den Heuvel M., Appel M., Sorop T., Wilson O.B., Berg S. (2021) Capillarity and phase-mobility of a hydrocarbon gas-liquid system, Oil Gas Sci. Technol. - Rev. IFP Energies nouvelles 76, 43.

4 Andersen P.Ø. (2021) Analytical modeling and correction of steady state relative permeability experiments with capillary end effects - An improved intercept method, scaling and general capillary numbers, Oil Gas Sci. Technol. - Rev. IFP Energies nouvelles 76, 61 . 